\title{
Flexible Model for Miro-launcher Dynamics
}

\author{
Teodor-Viorel Chelaru ${ }^{\text {, }, ~ *, ~ V a l e n t i n ~ P a n a ~}{ }^{\text {, Alexandru Iulian Onel }}{ }^{2}$, Tudorel-Petronel \\ A filipoae ${ }^{2}$, Andrei Filip Cojocaru ${ }^{3}$, and Ionut Cosmin V asile ${ }^{I}$ \\ 1Universiry POLITEH NICA of B ucharest, Faculty of A erospace Engineering, Romania \\ ${ }^{2} \mathrm{~N}$ ational Institute for A erospace Research "Elie Carafoli" \pm IN CA S, R omania \\ ${ }^{3}$ Deimos Space Romania
}

\begin{abstract}
The paper presents aspects regarding flexible model used for describing the dynamics of the three stages micro-launcher. This work analyses transverse flexible oscillations. By the hypotheses adopted, the flexibility problem will be reduced to a group of equations that will be attached to the rigid body model with six degrees of freedom, thus obtaining an elastic model for the launcher. The results analysed will be the flight parameters the launcher, with the influence of the elastic modes considered. The novelty of the paper consists in highlighting the influence of elasticity on the launcher control problem.
\end{abstract}

\section{Introduction}

As shown in reference [1] and [2], the analysis of the structural dynamicity of a vehicle is a complex problem, which involves an approach in matrix technique, with the use of specialized codes like ART eM IS M odal [3]. A Iso, for analysis of the dynamic flight of the launch vehicle the complex code is necessary. In this regard in reference [4] are presented some solutions related to the design process of flight vehicles using ASTOS code. In addition, is necessary to addressed al so the flight control problem. As an example, in work [5] is described the principles of launch vehicle flight control with application for A res I. Linking these two items is more difficult, the most common approach being to transfer the results of structural analysis to the flight control issue. To avoid this development, we are still proposing to find a simple analytical solution to the problem of transvers elastic oscillations of small-scale launchers that can easily be integrated into the developed dynamics code. The approach is based on solid fuel rocket work [6], but can al so be expanded to rocket with liquid fuel, if the structure is compact enough to be approximated with a homogeneous bar.

\section{Theoretical development}

As shown in the paper [6], in the assumption of the beam, the transvers oscillation of a launcher due to the elasticity of the body can be described with the differential equation:

$$
E I_{x} \frac{d^{2} w}{d x^{2}}=M(x, t)
$$

where $w=w(x, t)$ is the deformation of the elastic axis of the launcher, $E$ is the elasticity module of the material from which the launcher is composed, $I_{x}=\int z^{2} d A$ is the axial moment of inertia and is $M(x, t)$ the bending moment applied throughout the body, varying over time.

\footnotetext{
* Corresponding author: teodor.chelaru@upb.ro
} 
If we differentiate the relationship, results:

$$
\frac{\partial^{2}}{\partial x^{2}}\left(E I_{x} \frac{d^{2} w}{d x^{2}}\right)+m_{x} \frac{\partial^{2} w}{\partial t^{2}}=q(x, t)
$$

where $m_{x}$ represents the distribution of mass al ong the body and $q(x, t)$ the intensity of the external lateral load

\subsection{Free oscillations}

To obtain free oscillations, we consider the equation (2) in the homogenous form:

$$
\frac{\partial^{2}}{\partial x^{2}}\left(E I_{x} \frac{d^{2} w}{d x^{2}}\right)+m_{x} \frac{\partial^{2} w}{\partial t^{2}}=0
$$

for which free flight conditions are imposed:

$$
\left.m_{x} \frac{\partial^{2} w}{\partial x^{2}}\right|_{\substack{x=0 \\ x=l}}=0 ; \frac{\partial}{\partial x}\left(E I_{x} \frac{d^{2} w}{d x^{2}}\right)_{\substack{x=0 \\ x=l}}=0
$$

which represents the bending moment null and cutting force null. To obtain the equation solution, the initial form of the deformation and its first derivative should be known throughout the body:

$$
w(x, 0)=u(x) ; \frac{\partial w(x, 0)}{\partial t}=v(x) .
$$

which are the initial conditions of the problem. B ecause the body of the launcher will achieve harmonic oscillations, we will look for the solution of the homogeneous equation (3) with the conditions (4), (5)

$$
w(x, t)=\phi(x) \cos (\omega t+\varepsilon) .
$$

Entering the solution (6) in equation (3), we obtain:

$$
\left(E I_{x} \phi^{\prime \prime}\right)^{\prime \prime}-\omega^{2} m_{x} \phi=0,
$$

This equation covers all its own pulsations and forms of oscillation, of which we can choose own pulsation $k$ to look for the solution of the problem:

$$
\left(E I_{x} \phi^{\prime \prime}\right)^{\prime \prime}-\omega_{k}^{2} m_{x} \phi_{k}=0,
$$

with the conditions at the ends:

$$
\left.\frac{\partial^{2} \phi_{k}}{\partial x^{2}}\right|_{\substack{x=0 \\ x=l}}=0 ; \frac{\partial}{\partial x}\left(E I_{x} \frac{d^{2} \phi_{k}}{d x^{2}}\right)_{\substack{x=0 \\ x=l}}=0 .
$$

To find a simple solution to the problem, as shown in [6], we will consider a homogenous body of constant section. If the elastic modulus $E$ and the axial inertial moment $I_{x}$ no longer vary along the longitudinal axis, the equation ( 8 ) become:

$$
\phi^{I V}-\lambda^{4} \phi=0,
$$

where we denote:

with the solution:

$$
\lambda^{4}=\frac{m \omega^{2}}{E I},
$$

where $S(x), T(x), U(x), V(x)$ are K rilov functions:

$$
\phi(x)=A S(x)+B T(x)+C U(x)+D V(x),
$$

$$
\begin{aligned}
& S(x)=\frac{1}{2}(\operatorname{ch} \lambda x+\cos \lambda x) ; T(x)=\frac{1}{2}(\operatorname{sh} \lambda x+\sin \lambda x) ; \\
& U(x)=\frac{1}{2}(\operatorname{ch} \lambda x-\cos \lambda x) ; V(x)=\frac{1}{2}(\operatorname{sh} \lambda x-\sin \lambda x),
\end{aligned}
$$

and $A, B, C, D$ there are constant integration. Imposing conditions (9) at first end: 
two integration constants are null

$$
\phi_{\mid x=0}^{\prime \prime}=0 ; \phi^{\prime \prime \prime}{ }_{\mid x=0}=0,
$$

obtained, from where

$$
C=0 ; D=0,
$$

Imposing conditions (9) at second end:

$$
\phi(x)=A S(x)+B T(x)
$$

$$
\phi^{\prime \prime}{ }_{\mid x=l}=0 ; \phi^{\prime \prime \prime}{ }_{\mid x=l}=0,
$$

we obtain the following system with two unknowns $A, B$ :

$$
\phi^{\prime \prime}(l)=\lambda^{2}[A U(l)+B V(l)]=0 ; \phi^{\prime \prime \prime}(l)=\lambda^{2}[A T(l)+B U(l)]=0 \text {. }
$$

For the system (18) to not have only zero solutions, it is necessary that its determinant be null, from where:

$$
\cos \lambda l \times \operatorname{ch} \lambda l=1
$$

that means a transcendent equation with the following solution string:

$$
\lambda_{1} l=4,73, \lambda_{2} l=7,85, \underline{\circ} \lambda_{k} l \cong \frac{2 k+1}{2} \pi
$$

Replacing in the relationship (11) we obtain:

$$
\omega_{k} \cong \pi^{2}\left(\frac{2 k+1}{2 l}\right)^{2} \sqrt{\frac{E I}{m}}
$$

Further we can develop the previous assumptions, considering the body an empty cylinder, with a section of the shape of a circular crown. In this case from (21) we obtain:

$$
\omega_{k}=\frac{\pi^{2}}{2}\left(\frac{2 k+1}{2 l}\right)^{2} \sqrt{\frac{E}{\rho}\left(\frac{d^{2}}{2}-\frac{M}{\rho \pi l}\right)},
$$

where $\rho$ is material density, $M$ launcher mass; $l$ launcher length; $d$ body diameter.

N ext, we can evaluate the shape of the oscillation. For this, from relationships (18)

$$
\frac{B_{k}}{A_{k}}=-\frac{T_{k}(l)}{B_{k}(l)}=-\frac{U_{k}(l)}{V_{k}(l)}
$$

and from (16) it is obtained:

From (6)

$$
\phi_{k}(x)=A_{k}\left(S_{k}(x)-\frac{T_{k}(l)}{U_{k}(l)} T_{k}(x)\right)
$$

$$
w_{k}(x, t)=A_{k}\left(S_{k}(x)-\frac{T_{k}(l)}{U_{k}(l)} T_{k}(x)\right) \cos \left(\omega_{k} t+\varepsilon_{k}\right),
$$

where the initial phase $\varepsilon_{k}$ and the integration constant $A_{k}$ can be obtained from the initial conditions (5).

\subsection{Forced oscillations}

W hat we are still interested in is the influence of elastic transverse oscillations on a point of the launcher, which can be the position of guidance system or the position of payload. For this we return to the equation (2), which, in the hypothesis of a constant section body, acquires the form:

If we replace the relationship (6)

$$
E I \frac{d^{4} w}{d x^{4}}+m \frac{\partial^{2} w}{\partial t^{2}}=q(x, t)
$$

$$
E I \frac{d^{4} \phi}{d x^{4}} \cos (\omega t+\varepsilon)+m \frac{\partial^{2} w}{\partial t^{2}}=q(x, t)
$$

On the other hand, replacing the derivatives of four order of (10) 


$$
E I \lambda^{4} \phi \cos (\omega t+\varepsilon)+m \frac{\partial^{2} w}{\partial t^{2}}=q(x, t) .
$$

A fter processing, for the $k$ oscillation mode, it is obtained:

$$
\frac{\partial^{2} w_{k}}{\partial t^{2}}+\omega_{k}^{2} w_{k}=\frac{q(x, t)}{m}
$$

where $m$ is a constant mass distribution along the body.

If we consider that the source of the oscillations only the lateral component of the thrust force, its influence on a point on the launcher becomes:

$$
q(x, t)=\frac{z^{T}(l-x)}{l^{2}}
$$

where $x$ is the position at the peak of the launcher of the point where we evaluate the deformation, $l$ is the body length. The lateral component of the thrust:

$$
Z^{T}=T \sin \delta_{m}(t),
$$

depends on time.

From (29), taking into account that $M=m l$, we obtain:

$$
\ddot{w}_{k}+\omega_{k}^{2} w=\frac{T(l-x)}{M l} \sin \delta_{m}
$$

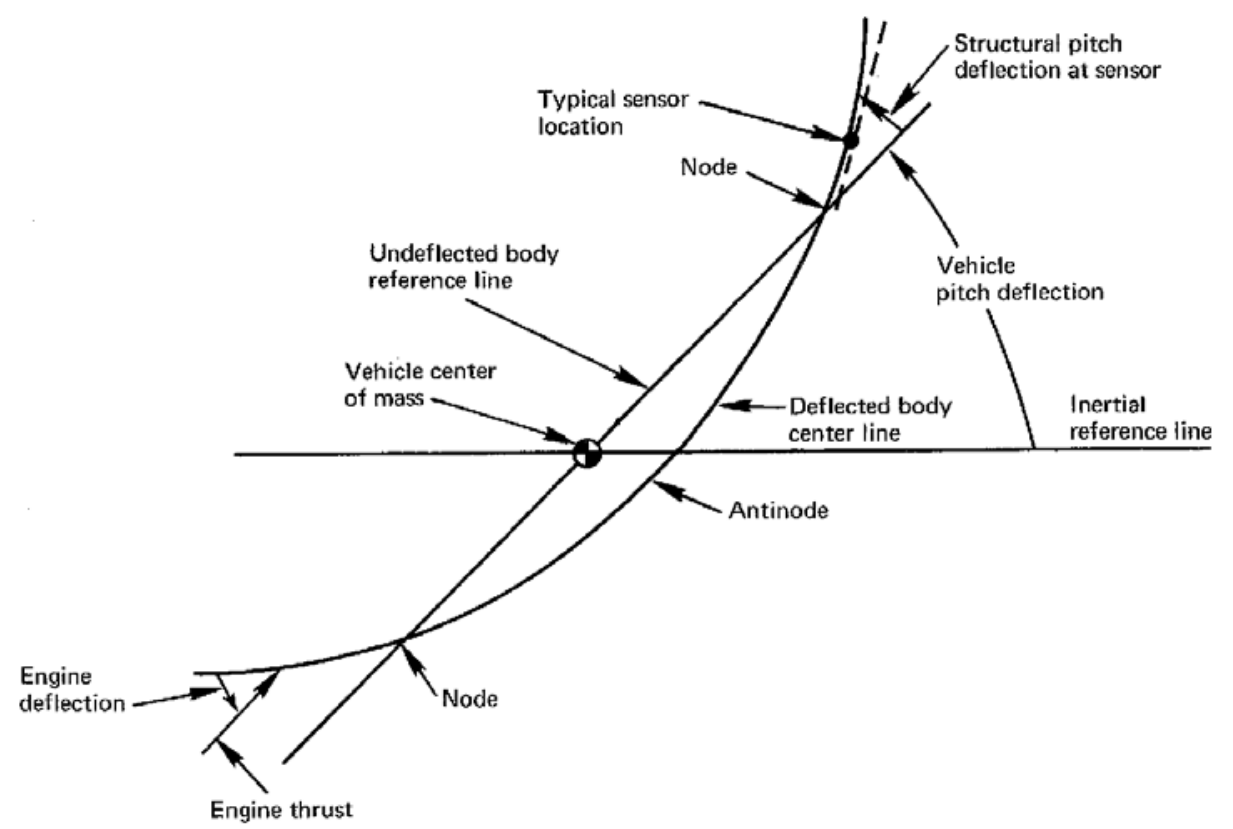

Fig. 1 Idealized flexible launch vehicle [2]

If we transform the linear deformation into angular deviation, we obtain:

$$
\ddot{\psi}_{k}+\omega_{k}^{2} \psi=K_{S} \sin \delta_{m} \text {. }
$$

where we denoted the gain:

$$
K_{S}=\frac{T(l-x)}{M l^{2}} .
$$

As shown in works [2] [7] [1], in addition to the considered model the oscillations of the launcher have damping effect, the equation (33)

$$
\ddot{\psi}_{k}+2 \xi_{k} \omega_{k} \dot{\psi}_{k}+\omega_{k}^{2} \psi=K_{S} \sin \delta_{m}
$$

where, as shown in work [2] the damping coefficient, has values ranging between: 


$$
\xi_{k}=0,005 \ldots 0,02 \text {. }
$$

If we retain the first $n$ frequency, then from (35)

$$
\dot{q}_{k}=-2 \xi_{k} \omega_{k} q_{k}-\omega_{k}^{2} \psi+K_{S} \sin \delta_{m} ; \dot{\psi}_{k}=q_{k} \text {, where } k=1, n
$$

The angle and angular velocity in the oscillation plane will be affected by the sum of the deviations introduced by the elastic oscillations:

$$
q *=q+\Delta q ; \psi *=\psi+\Delta \psi
$$

where $q, \psi$ are the actual values, $q *, \psi *$ are the measured values and $\Delta q, \Delta \psi$ are the influence of the flexible oscillations of the launcher structure in yaw.

$$
\Delta q=\sum_{k=1}^{n} q_{k} ; \Delta \psi=\sum_{k=1}^{n} \psi_{k}
$$

\section{Input data for micro-launcher model}

The main input data used are extracted from paper [8]. In Fig. 2 we have: P/L Payload; ST Stage; All sizes are in meters.
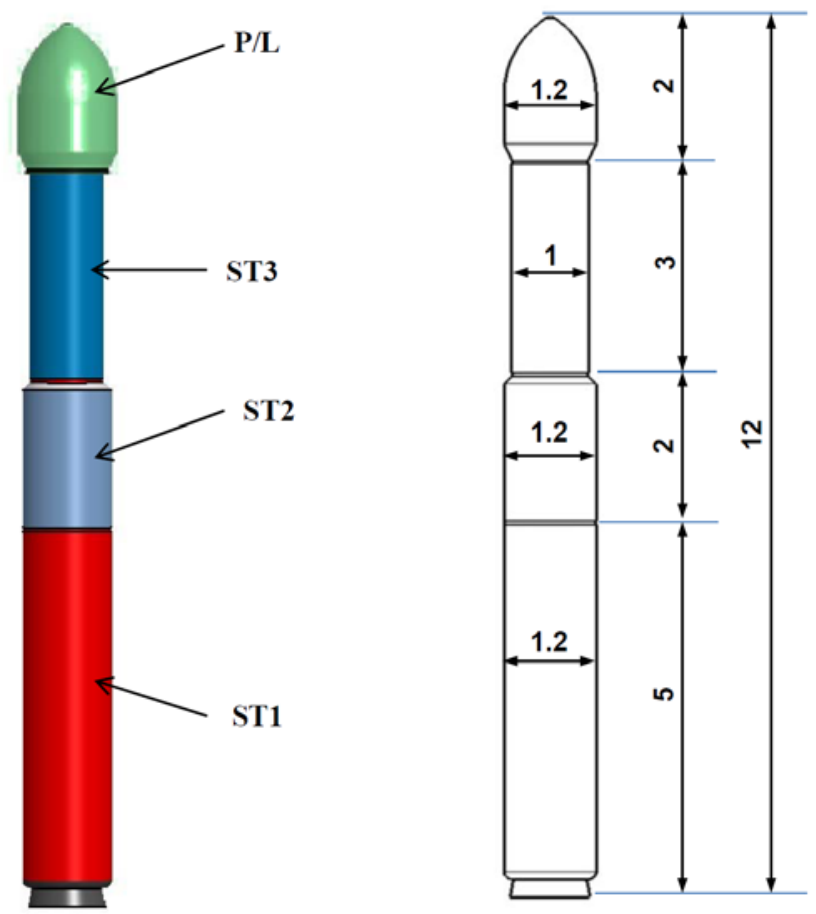

Fig. 2 M icro-launcher configuration [8]

\section{Results}

Using relation (22) we obtain first three own frequencies, shown in Fig. 3. One can observe that during launcher evolution the own frequencies increases due to the fact that the launcher size is decreasing. The values obtained are close to those indicated in the paper [2]. 


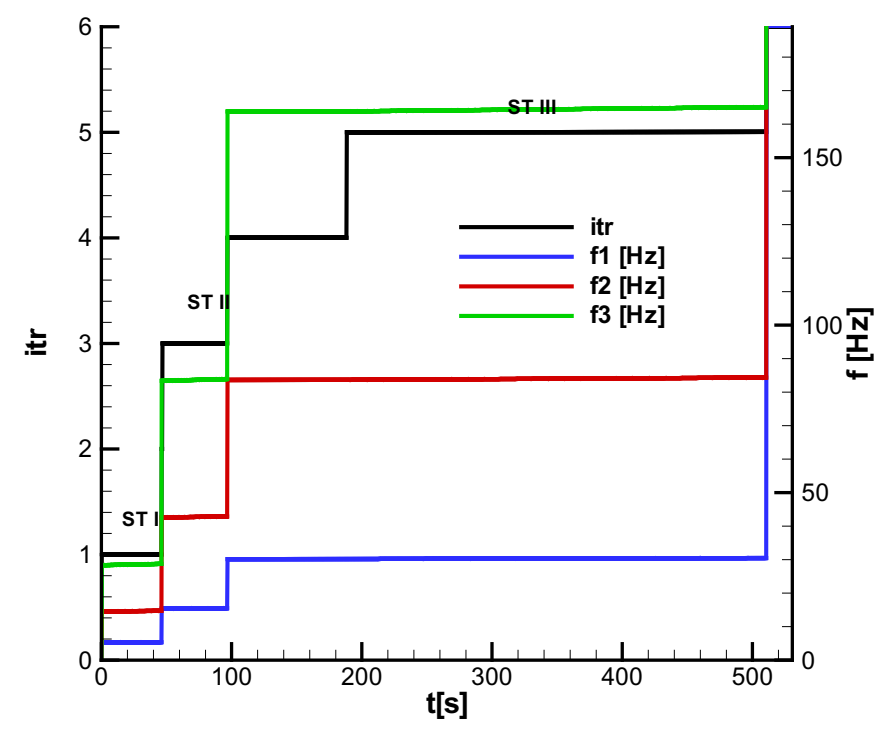

Fig. 30 wn frequencies during launcher evolution

U sing relation (34) we obtain the gain $K_{S}$ for transverse flexible oscillation shown in Fig. 4. One can observe that during launcher evolution the gain follow the thrust values.

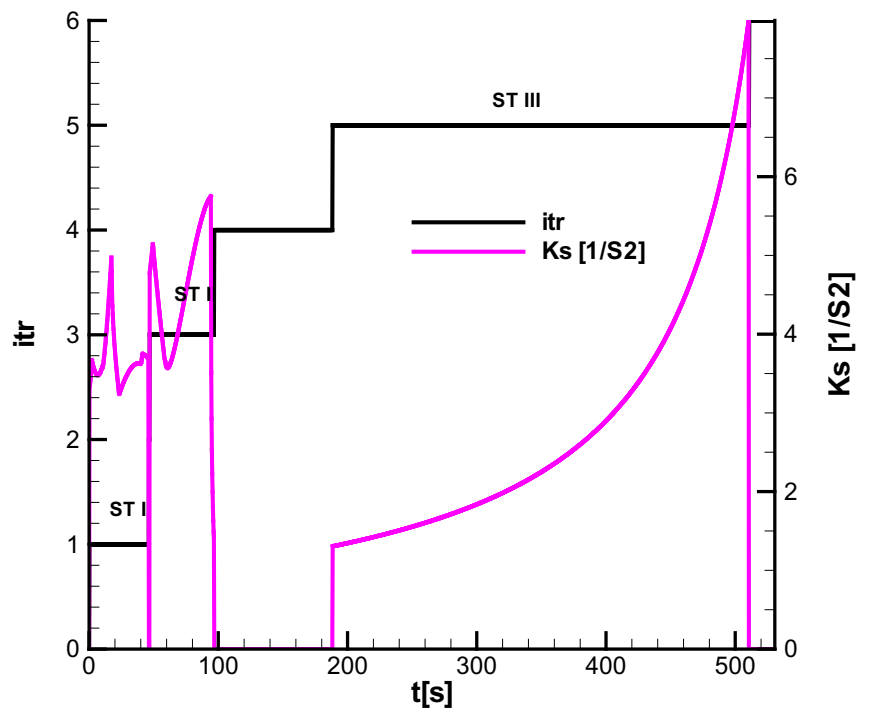

Fig. 4 Gain for flexible oscillations

B ased on relation (39) we obtain the influence of flexible oscillation on pitch angle (DTE) and yaw angle $(D P S)$ shown in Fig. 5 . On can observe that these angular deviations follow the angular deflections of the thrust vector $\delta_{n}, \delta_{m}$. 


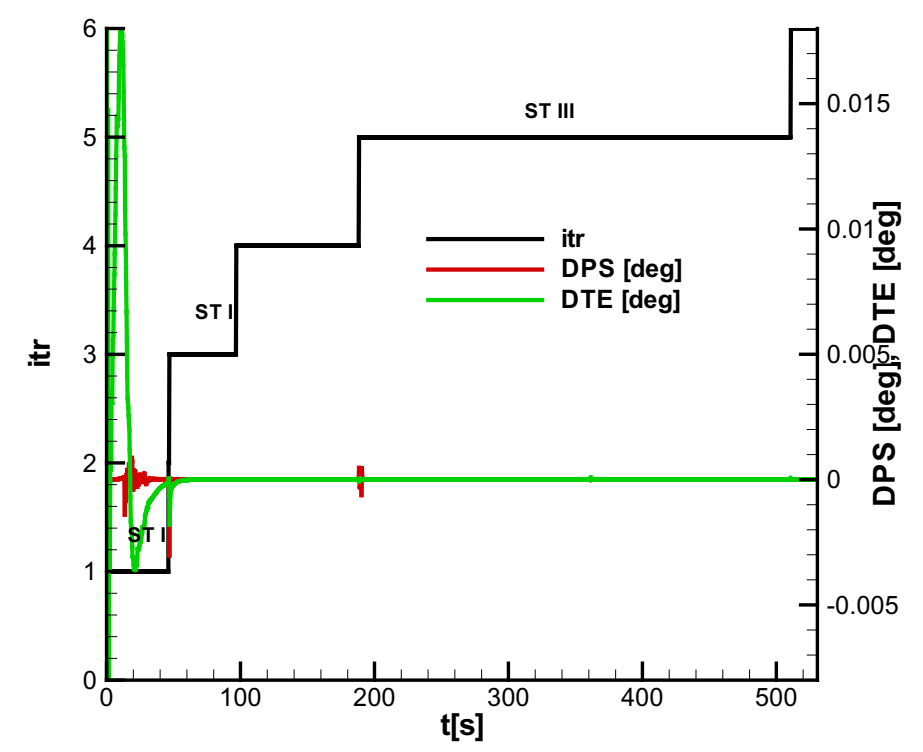

Fig. 5 A ngular deviation due to flexible modes in the guidance section

B ased also on relation (39) we obtain the influence of flexible oscillation on pitch angle velocity $(D r)$ and yaw angle velocity $(D q)$ shown in Fig. 6. On can observe that these angular velocity deviations follow also the angular deflections of the thrust vector $\delta_{n}, \delta_{m}$.

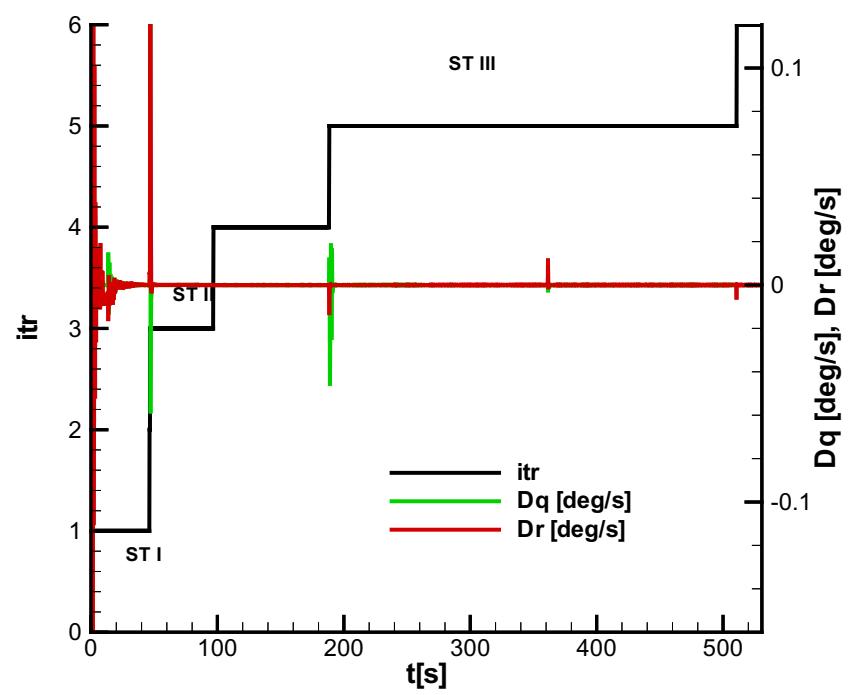

Fig. 6 A ngular velocity deviations due to flexible modes in the guidance section

\section{Conclusions}

As stated in the introduction the work seeks to highlight the influence of transversal oscillations of the structure on the control of the launcher. For this, simple relationships have been obtained for the calculation of own pulsations and gain necessary to construct the equations of flexible modes, which can be easily attached to the rigid model with six degrees of freedom (6DOF). B ased on the flexible model developed, were evaluated the deviations in assessing the main control sizes: angles and velocity angle in the yaw and pitch, that can 
be considered in the synthesis of the launcher control system. However, it is noted from the results obtained that the errors introduced by the flexible modes in the measurement of angles and angle velocity are not very high, compensation can be made by increasing the robustness of the control system of the launcher. The analytical results obtained, beside the integration into the dynamic code, can be used in other applications, for a preliminary evaluation of the launcher features.

In order to obtain an analytical simplified results in this paper we approach only transvers oscillations. Following similarly technique, in future works we will approach also longitudinal oscillations.

\section{References}

[1] A. Petre, Calculul structurilor de aviatie, Bucuresti: E ditura Tehnica, (1984).

[2] R. B. Noll, J. Zvara, J. J. D eyst, "Effects of structural flexibility on launch vehicle control systems," NASA, Sprinfield, V irginia, (1970).

[3] xxx, "http://www.svibs.com/index," [Online].

[4] A. Wiegand, F Cremaschi, S. W inter, S W eikert, "D esign Optimization and performance analysis of launch vehicles with ASTOS," D eutscher L uft-und Raumfahrtkongress, 2014.

[5] B. Wie, W. Du, M. Whorton, "A nalysis and D esign of L aunch V ehicle Flight Control Systems," NASA, 2008.

[6] А.М. Синюков, Л.И. Болков, А.И. Львов, А.М. Шишкевич, Баллистическая ракета на твердом топливе, Москва: Издательство Министерства Обороны СССР, (1972).

[7] A. H. E sbati, S. Irani, "Structural dynamic stability of three stages launch vehicle as a free-free Timoshenko beam subjected to follower force," in Recent Advances in Space Technologies $R A S T$, Istanbul, (2009).

[8] T.V. Chelaru, "Performances Evaluation for M icrolauncher, M athematical M odel," International Journal of Modeling and Optimization,, vol. 8, no. 4, pp. 197-201, Aug (2018). 